\title{
Disordered-surface-layer transition in nematic liquid crystals
}

\author{
Ping Sheng, Bo-Zang Li, ${ }^{*}$ and Minyao Zhou \\ Exxon Research and Engineering Company, Route 22 East, Clinton Township, Annandale, New Jersey 08801
}

Thomas Moses and Y. R. Shen

Department of Physics, University of California, Berkeley, California 94720

(Received 1 April 1992)

\begin{abstract}
A disordered nematic surface layer, with negative orientational order or biaxial order, is shown to result from interaction with substrates which favor random planar alignment. We propose a simple interaction Hamiltonian which yields predictions in excellent agreement with measured pretransitional effects for 4- $n$-pentyl-4'-cyanobiphenyl on clean glass and unrubbed $n$-methylaminopropyltrimethoxysilane substrates. When the dimensionless interaction strength is larger than a critical value, a pretransitional biaxial boundary layer is predicted for a temperature range which is bounded above by surface biaxial-uniaxial and biaxial-biaxial transitions, and below by a complete wetting transition of the boundary layer.
\end{abstract}

PACS number(s): 61.30.Cz, 64.70.Md, 68.45.-v

As an integral part of the liquid-crystal display technology, substrate alignment of liquid crystals is a subject of both practical and basic scientific interest. For substrates treated by rubbing or coating techniques, various theoretical and experimental studies in the past two decades have revealed the existence of a surface layer [1-6], on the order of $100-1000 \AA$, which transmits the aligning action of the substrate to the bulk as well as controls the wetting property of the liquid crystals [7]. For nematic liquid crystals, the surface layer on a treated substrate has been found to have phase-transition characteristics distinct from those of the bulk $[1,3,5]$. In this work, we show that for an untreated substrate with no particular aligning effect the surface layer can present a new set of phenomena. In particular, for nematic liquid crystals whose molecules prefer to lie perpendicular to the substrate normal, the surface layer not only has negative orientational order, but can also have a first-order director orientational transition, as well as a uniaxial-biaxial surface transition when the interaction strength exceeds a critical value. Experimentally measured pretransitional effect shows excellent agreement with theoretical predictions.

Consider a nematic-liquid-crystal sample in the half space $z \geq 0$ with an order parameter defined by [8] $Q_{i j}=S\left(n_{i} n_{j}-\delta_{i j} / 3\right)$, where $S=\left\langle P_{2}(\cos \theta)\right\rangle, P_{2}$ denotes the second-order Legendre polynomial, $n_{i}$ the $i$ th component of the director unit vector $\hat{\mathbf{n}}, \theta$ the angle between the molecular axis and $\hat{\mathbf{n}}$, and the angular brackets denotes volume averaging. The lowest-order substrate-nematic-liquid-crystal interaction at an isotropic surface has the form $H_{s}=G \widehat{\mathbf{k}} \cdot \mathbf{Q} \cdot \hat{\mathbf{k}} \delta(z)$, where $\widehat{\mathbf{k}}$ is the unit substrate normal and $G$ denotes the interaction strength. We will use $\hat{\mathbf{i}}, \hat{\mathbf{j}}$, and $\hat{\mathbf{k}}$ to denote the unit vectors in three orthogonal directions in the laboratory frame. For $G$ negative, this interaction with $S>0$ implies a homeotropic aligning substrate-nematic-liquid-crystal interaction whose implications have been studied before
[1-6]. In this work, we show that for $G$ positive the interaction can also denote the effect of a nonaligning substrate on which the molecules prefer to lie perpendicular to $\hat{\mathbf{k}}$. In that case, since the director $\hat{\mathbf{n}}$ is defined as the axis of uniaxial symmetry, the favored state is where the molecular axes point randomly in the plane of the substrate so that $\hat{\mathbf{n}} \| \hat{\mathbf{k}}$ but $S$ is negative [8], which makes the interaction energy also negative. However, the possibility of spontaneous symmetry breaking in the plane of the substrate means that one must consider a biaxial order parameter [9] near the substrate, i.e., $Q_{i j}=S\left(n_{i} n_{j}-\delta_{i j} / 3\right)+P\left(l_{i} l_{j}-m_{i} m_{j}\right)$, where $\hat{1} \perp \hat{\mathbf{n}}$ and is in the plane formed by $\hat{\mathbf{n}}$ and $\hat{\mathbf{k}}$, $\hat{\mathbf{m}}$ is perpendicular to both $\hat{\mathbf{1}}$ and $\hat{\mathbf{n}}$, and $P$ is the additional order parameter, representing the asymmetry of the molecular state in the $\hat{\mathbf{1}}, \hat{\mathbf{m}}$ plane, associated with a biaxial state. Therefore, $H_{s}=G \delta(z)\left\{S\left[(\widehat{\mathbf{n}} \cdot \hat{\mathbf{k}})^{2}-\frac{1}{3}\right]+P(\hat{\mathbf{k}} \cdot \hat{\mathbf{1}})^{2}\right\}$ is the complete form of the substrate-nematic-liquid-crystal interaction if we assume $\hat{\mathbf{n}}$ to be in the plane formed by $\hat{\mathbf{k}}$ and $\hat{\mathbf{l}}$. Below we show that such a substrate-nematic-liquid-crystal interaction can induce a pretransitional surface layer of negative order or biaxial order, as well as surface uniaxial-biaxial transition, biaxial-biaxial transition, and a complete wetting transition by the boundary layer.

For mathematical simplicity, in this work we follow the Sluckin-Poniewierski model [9] for the biaxial nematic state. In the Landau-de Gennes formalism, the total free-energy density, including the possibility of biaxiality, is given by $[9,10]$

$$
\begin{aligned}
f= & p(S, P, T)+L\left[\frac{d S}{d z}\right]^{2} \\
+G \delta(z) & {\left[(S-P) \cos ^{2} \psi+\left[P-\frac{S}{3}\right]\right]+H_{\varepsilon}, } \\
p(S, P, T)= & a\left(T-T^{*}\right)\left(S^{2}+3 P^{2}\right)-B S\left(S^{2}-9 P^{2}\right) \\
& +C\left(S^{2}+3 P^{2}\right)^{2}
\end{aligned}
$$


where $\psi$ is the angle between $\hat{\mathbf{n}}$ and $\hat{\mathbf{k}}, T^{*}$ is the minimum supercooling temperature (also called the virtual transition temperature), $a, B, C$, and $L$ are the material constants, and $H_{\varepsilon} \sim-\varepsilon \delta(z) S \sin ^{2} \psi \cos ^{2}\left(\phi-\phi_{0}\right)$, with $\varepsilon \rightarrow 0^{+}$, is an infinitesimal symmetry-breaking potential intended to lift the degeneracy in the $x-y$ plane, with $\phi_{0}$ denoting the symmetry-breaking direction.

Equation (1) is derived under two assumptions. First, the angle $\psi$ for the bulk state is assumed to be either 0 or $\pi / 2$. At these two values of $\psi$, it can be shown that $d \psi / d z=0$ and $d^{2} \psi / d z^{2}=0$ identically, and $\psi$ is therefore a constant throughout the sample. Second, the terms containing $(d P / d z)$ are dropped so that the theory becomes analytically integrable. Analysis of these two assumptions has shown that the overall qualitative behavior of the original model is most likely to be preserved [10].

For the purpose of calculation, it is more convenient to express the $Q_{i j}$ in laboratory coordinates. By assuming that $\hat{\mathbf{m}} \| \hat{\mathbf{j}}$, we have

$$
\mathbf{Q}=U\left(\hat{\mathbf{k}} \hat{\mathbf{k}}-\frac{\mathbf{I}}{3}\right)+V(\hat{\mathbf{i}}-\widehat{\mathbf{j}} \hat{\mathbf{j}}),
$$

where

$$
\begin{aligned}
& U=\left\{\begin{array}{l}
S, \quad \psi=0 \\
\frac{3}{2} P-\frac{1}{2} S, \quad \psi=\frac{\pi}{2},
\end{array}\right. \\
& V= \begin{cases}P, \quad \psi=0 \\
\frac{1}{2}(S+P), \quad \psi=\frac{\pi}{2},\end{cases}
\end{aligned}
$$

and $I$ denotes the identity matrix. The form of $p$ is invariant under this transformation. This is,

$$
\begin{aligned}
p(U, V, T)= & a\left(T-T^{*}\right)\left(U^{2}+3 V^{2}\right)-B U\left(U^{2}-9 V^{2}\right) \\
& +C\left(U^{2}+3 V^{2}\right)^{2}
\end{aligned}
$$

In dimensionless form, the free energy to be minimized is that induced by the surface, which may be expressed as

$$
F=\frac{2}{3} g u_{s}+\int_{0}^{\infty} d \xi\left[p(u, v, t)-p(u, 0, t)+\left[\frac{d u}{d \xi}\right]^{2}\right]
$$

Here $u=C U / B, \quad v=C V / \underline{B}, \quad \xi=B z / \sqrt{C L}, \quad t=a C(T$ $\left.-T_{K}^{0}\right) / B^{2}, g=C^{3 / 2} G / B^{2} \sqrt{L}, T_{K}^{0}=T^{*}+B^{2} / 4 a C$ is the bulk nematic-isotropic transition temperature, $u_{B}$ is the bulk nematic order parameter, given by

$$
u_{B}=\left\{\begin{array}{l}
\frac{1}{8}(3+\sqrt{1-32 t}), \quad t \leq 0 \\
0, \quad t>0
\end{array}\right.
$$

and $u_{s}$ denotes the order parameter value at the substrate, $\xi=0$. The expression for $u_{B}$ is obtained as the absolute minimum of the dimensionless $p(u, 0, t)$ :

$$
p(u, 0, t)=\left(t+\frac{1}{4}\right) u^{2}-u^{3}+u^{4} .
$$

The appearance of $p\left(u_{B}, 0, t\right)$ in Eq. (3) is due to the fact that the surface part of the free energy is defined as the difference between the total free energy and the bulk free energy, which is given by the integral of $p\left(u_{B}, 0, t\right)$. For the liquid crystal 4- $n$-pentyl-4'-cyanobiphenyl (5CB) [11], $a \simeq 0.065 \mathrm{~J} \mathrm{~cm}{ }^{-3} \mathrm{~K}^{-1}, B=0.53 \mathrm{~J} / \mathrm{cm}^{3}, C=0.98 \mathrm{~J} / \mathrm{cm}^{3}$, and $L \simeq 4.5 \times 10^{-14} \mathrm{~J} / \mathrm{cm}$, which implies that $\Delta t=1$ translates into $\Delta T \simeq 4 \mathrm{~K}, \Delta \xi=1$ translates into $\Delta z \simeq 40$ $\AA$, and $\Delta u, \Delta v=1$ means $\Delta S, \Delta P \simeq 0.5$.

It should be noted here that in terms of $u$ and $v$, there are two bulk states at $t<0$ with identical bulk free-energy densities. For the bulk state with $\hat{\mathbf{n}} \| \hat{\mathbf{k}}$, we have $v=0$ and $u=u_{B}$ given by Eq. (4a). For bulk state with $\hat{\mathbf{n}} \perp \hat{\mathbf{k}}$, on the other hand, we have $u=-u_{B} / 2$ and $v=u_{B} / 2$. These two bulk states pose different conditions at infinity for the boundary layer and therefore induce different free energies as calculated by Eq. (3) for $t<0$.

To minimize $F$, we first note that the Euler-Lagrange equation for the variable $v$ results in an equation which may be solved analytically to get $v$ in terms of $u$. The result is

$$
v=\left\{\begin{array}{l}
{\left[\frac{\left(u-u_{-}\right)\left(u_{+}-u\right)}{3}\right]^{1 / 2}, \quad u_{-} \leq u \leq u_{+}} \\
0, \text { otherwise },
\end{array}\right.
$$

where

$$
u_{ \pm}=\frac{-3^{ \pm} \sqrt{9-8(t+1 / 4)}}{4}
$$

and $v=0$ for $t \geq 7 / 8$. Below we will write $p(u, v, t)$ as $p(u, t)$. As a function of $u, p(u, t)$ has three minima for $-\frac{1}{4} \leq t \leq \frac{1}{32}$ at $u=u_{B}, 0$, and $-u_{B} / 2$. In the range of $0<t, u=0$ is the absolute minimum. For $t \leq 0, u=u_{B}$, $v=0$, and $u=-u_{B} / 2, v=u_{B} / 2$ are equivalent uniaxial states with the same lowest free energy, but with the uniaxial axis either along the $\hat{\mathbf{k}}$ or the $\hat{\mathbf{i}}$ axes, respectively, as noted above. The first integral of the Euler-Lagrange equation for variable $u$ gives

$$
\frac{d u}{d \xi}=\operatorname{sgn}\left(u_{B}-u_{s}\right) \sqrt{p(u, t)-p\left(u_{B}, t\right)} .
$$

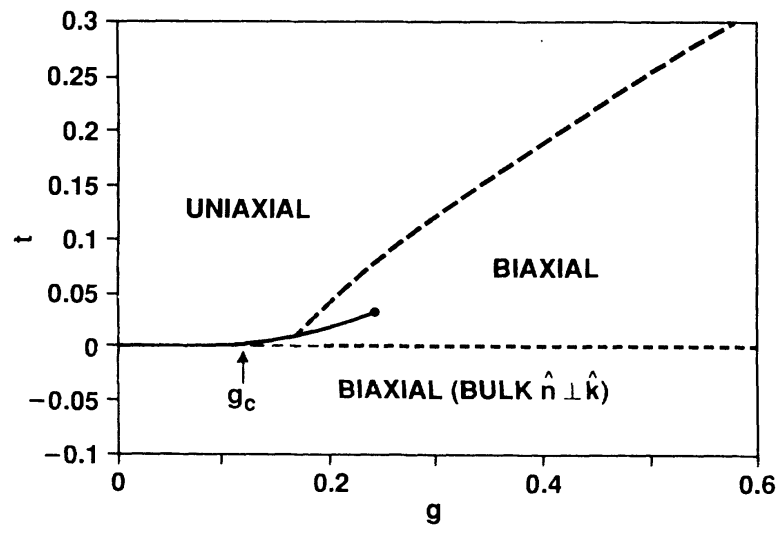

FIG. 1. Boundary-layer phase diagram as a function of $t$ and $g$. The solid line denotes a first-order phase transition. The dashed line denotes a second-order phase transition. 
By substituting Eq. (6) into Eq. (2), one gets

$F=\frac{2}{3} g u_{s}+2 \operatorname{sgn}\left(\tilde{u}_{B}-u_{s}\right) \int_{u_{s}}^{\tilde{u}_{B}} \sqrt{p(u, t)-p\left(u_{B}, t\right)} d u$.

Here $\widetilde{u}_{B}=u_{B}$ for $\hat{\mathbf{n}} \| \hat{\mathbf{k}}$, and $\widetilde{u}_{B}=-\widetilde{u}_{B} / 2$ for $\widehat{\mathbf{n}} \perp \hat{\mathbf{k}}$. Equation (7) is to be minimized with respect to $u_{s}$ and the two possible bulk states at $\xi=\infty$.

The two branches of minimum free-energy solutions, corresponding to $\psi=0, \pi / 2$, have been examined. The phase behavior obtained by the condition of minimum $F$ is shown in Fig. 1, where it is seen that there are basically two types of surface phases in the bulk isotropic tempera- ture range, $t \geq 0$, with both a first-order transition line, denoted by the solid line, and a second-order transition line, denoted by the dashed line, separating the different phases. For $g<g_{c}=0.12$ the boundary layer above $t_{c}=0$ is uniaxial with $u_{s}<0, v=0$. Below $t_{c}=0$ the bulk state with $\psi=\pi / 2$ is favored, and there is always a biaxial boundary layer. This situation is illustrated in Fig. 2 with $g=0.11$, where the biaxial boundary layer is seen to be $\sim 50 \AA$.

In the $g<g_{c}$ range, the surface state at $t \geq 0$ is always uniaxial $(v=0)$, and the spatial dependence of $u(\xi)$ can be explicitly obtained by integrating Eq. (6) once $u_{s}$ is known:

$$
\begin{aligned}
& u(\xi)=u_{B}-\frac{2 \beta A}{\alpha A-\sqrt{\beta} A^{2} \exp (\sqrt{\beta} \xi)+\left(\sqrt{\beta}-\alpha^{2} / 4 \sqrt{\beta}\right) \exp (-\sqrt{\bar{\beta}} \xi)}, \\
& \alpha=4 u_{B}-1 \text {, } \\
& \beta=6 u_{B}^{2}-3 u_{B}+t+\frac{1}{4} \text {, } \\
& A=\frac{\sqrt{\beta}+\left[\left(u_{s}-u_{B}\right)^{2}+\alpha\left(u_{s}-u_{B}\right)+\beta\right]^{1 / 2}}{u_{s}-u_{B}}+\frac{\alpha}{2 \sqrt{\beta}} .
\end{aligned}
$$

Equation (8) predicts a certain pretransitional behavior induced by the disordered surface layer $\left(\psi=0\right.$ and $\left.u_{s}<0\right)$. This part has been studied experimentally, using the evanescent-wave ellipsometry technique [7]. The quantity measured was the phase shift $\Delta \phi_{c}$ between the $p$ - and $s$-polarized components of a He-Ne laser beam $(\lambda=633$ $\mathrm{nm})$ totally reflected from a glass-liquid-crystal interface at the critical angle $\theta_{c}$. The sample cell was made by sandwiching a $130-\mu \mathrm{m}$ 5CB film between a glass prism (Schott glass LaSF 9 with a refractive index $n=1.84$ at $633 \mathrm{~nm}$ ) and a glass plate. The prism surface in contact with 5CB was either acid cleaned or acid cleaned and coated with a layer of $n$-methylaminopropyltrimethoxysilane (MAP) which was not rubbed. The cell was filled by capillary action with $5 \mathrm{CB}$ in the isotropic phase and loaded into an oven with a $\pm 1-\mathrm{mK}$ temperature stability. The laser beam was incident from the prism side at $\theta_{c}$ and the totally reflected beam was analyzed. It can be shown that the observed phase shift is directly proportional to the integrated birefringence $\gamma$ at

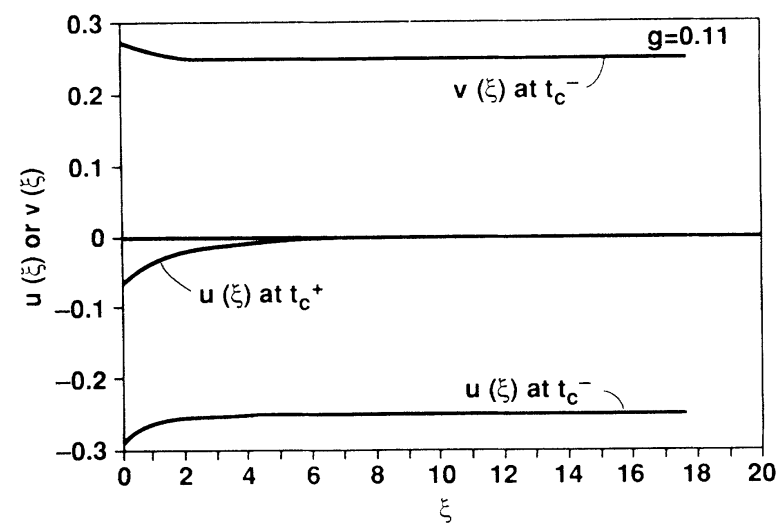

FIG. 2. Spatial variation of the order parameters for $g=0.11$ at $t_{c}^{+}=10^{5}$ and $t_{c}^{-}=-10^{-4}$. At $t_{c}^{+}, v(\xi)=0$ identically.

the interface

$$
\begin{aligned}
& \Delta \phi_{c}=2 \kappa \sqrt{L / C} \gamma \\
& \gamma=\int_{0}^{\infty} d \xi\left[u(\xi)-u_{B}\right]=\ln \left|\frac{\alpha-2 \sqrt{\beta}(1+A)}{\alpha+2 \sqrt{\beta}(1-A)}\right|,
\end{aligned}
$$

where $\kappa=3.37 \times 10^{-3} \AA^{-1}$ is an experimentally deter-

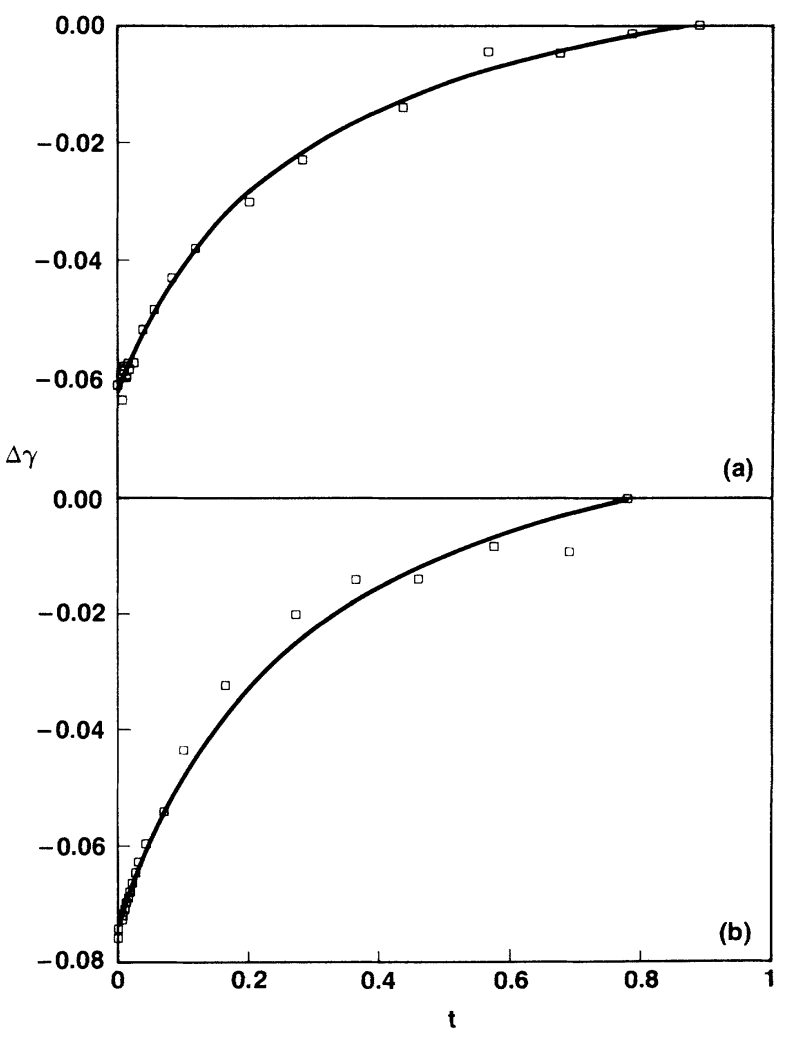

FIG. 3. Temperature variation of $\Delta \gamma$. Open squares denote data. The solid lines represent the one-parameter fits using Eq. (9). (a) Clean glass substrate. (b) Unrubbed MAP substrate. 


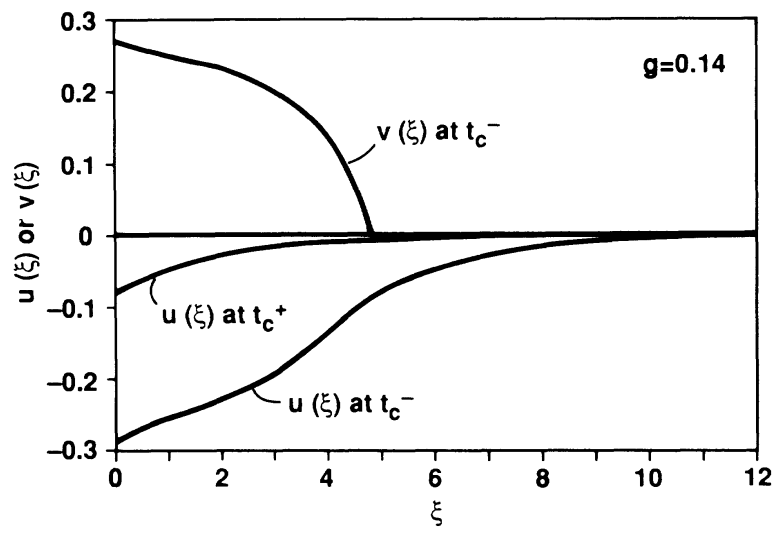

FIG. 4. Spatial variation of the order parameters for $g=0.14$ at $t_{c}=0.0026$. At $t_{c}^{-}=t_{c}-10^{-6}$, the boundary layer is biaxial. At $t_{c}^{+}=t_{c}+10^{-6}$, the boundary layer is uniaxial with $v=0$ identically.

mined proportional constant.

For each data run, $\Delta \phi_{c}$ was measured at a series of temperatures monotonically approaching the isotropic to nematic transition at $T_{K}^{0}$ from above. Sufficient time ( $>20 \mathrm{~min}$ ) was allowed for the sample to reach equilibrium at each temperature. The bulk phase transition could be clearly recognized by the sudden increase in scattered light and change in the critical angle. All data runs were taken within two days of the assembly of the cell in order to minimize the effects of migration of impurities into the interfacial region. The data were found to be highly reproducible. The error in $\Delta \phi_{c}$ was due primarily to system drift, which was no more than $0.5 \mathrm{mrad} / \mathrm{day}$.

Two sets of experimental data were taken. One is for $5 \mathrm{CB}$ on clean glass, and the other is on unrubbed MAPcoated glass. They are shown in Figs. 3(a) and 3(b) as $\Delta \gamma=\gamma-\gamma_{0}$, where $\gamma_{0}$ is the value of $\gamma$ at the highest measured temperature. The solid lines are the oneparameter $(g)$ theoretical fits using Eq. (8). The fittings yield $g=0.033$ for the clean glass and $g=0.036$ for the MAP-coated substrate. The good agreement between theory and experiment clearly shows that (1) $u_{s}$ and the surface layer have negative order parameter, indicating a

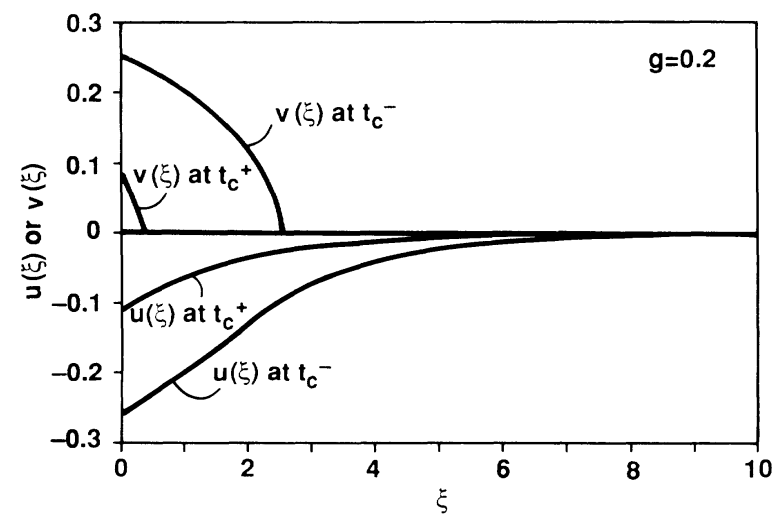

FIG. 5. Spatial variation of the order parameter for $g=0.2$ at $t_{c}=0.01645$. Both $u(\xi)$ and $v(\xi)$ experience first-order transitions. dewetting tendency and (2) the surface layer thickness increases as $t \rightarrow t_{c}$ from above so that $\gamma$ becomes more negative. The fact that $\gamma$ stays finite shows that the dewetting is only partial. In both cases, $g<g_{c}$ so that $t_{c}$ coincides with the bulk transition.

The transition at $t_{c}=0$ has been noted to involve a director orientational transition from $\psi=0$ to $\psi=\pi / 2$. This part of the theoretical prediction has also been verified by experiment since below $t_{c}$, a multidomain structure, each with director parallel to the substrate, was observed. This is in contrast to the case if the director were to remain perpendicular to the substrate, which would imply homeotropic alignment.

At $g=g_{c}=0.12$, the first-order transition line starts to deviate from $t=0$, and it terminates at a critical point with $g=0.24$ and $t=0.03$. For $g_{c}<g<0.163$, the firstorder transition separates the uniaxial boundary layer at $t>t_{c}$ from the biaxial boundary layer at $t<t_{c}$. This is illustrated in Fig. 4 for $g=0.14$. At $g \simeq 0.163$ and $t \approx 0.007$, an additional second-order transition line emerges on top of the first-order transition line. Therefore, for $0.163<g<0.24$ the boundary layer at $t>0$ has two transitions. The lower temperature one is first order and separates one biaxial boundary state from another. This is illustrated in Fig. 5 for $g=0.2$. The highertemperature one is second order and separates the uniaxial boundary state from the biaxial state. This secondorder transition involves $v$ only. The variation of $u$ is smooth across the transition. Beyond $g=0.24$ only the second-order transition persists.

All the rich behaviors described above can be summa-

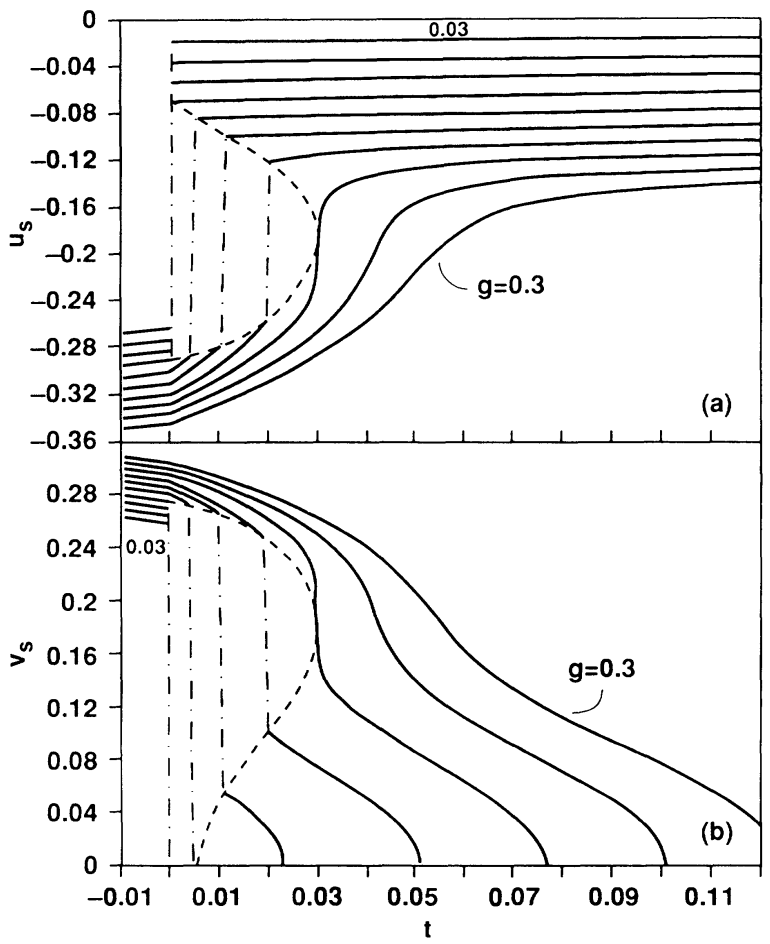

FIG. 6. Variation of $u_{s}$ and $v_{s}$ as a function of $t$, for $g$ varying from 0.03 to 0.3 in increments of 0.03 . The dashed lines delineate the coexistence curves of $u_{s}$ and $v_{s}$. 


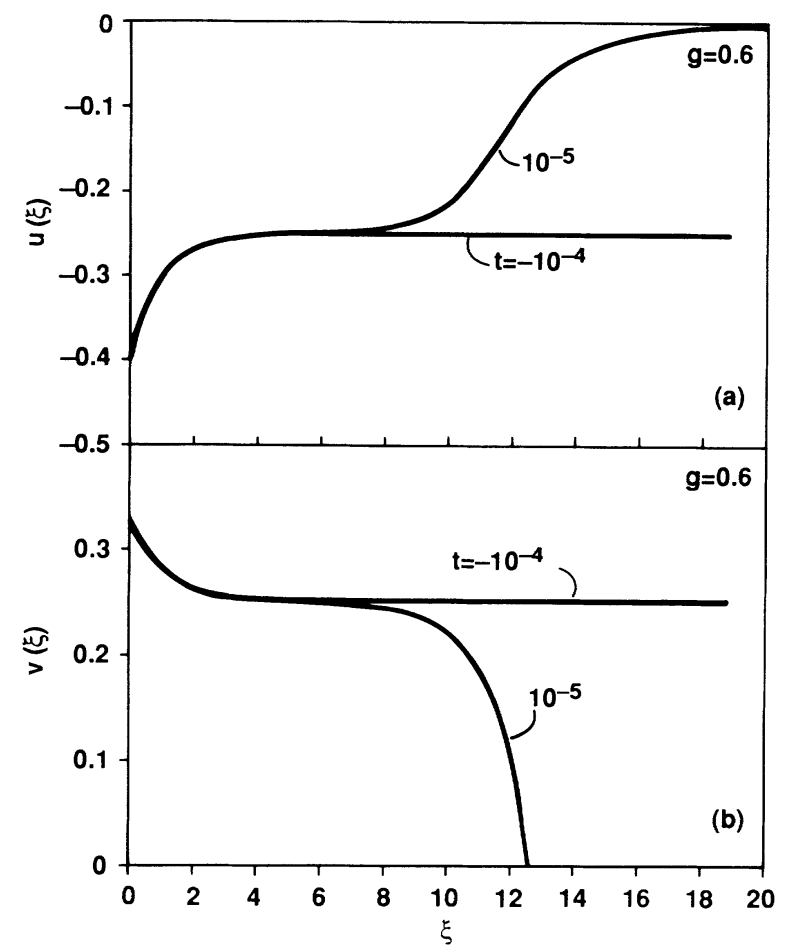

FIG. 7. Spatial variation of $u$ and $v$ at $t=10^{-5}$ and $-10^{-4}$. The flat portions of the order parameters at $t=10^{-5}$ indicate an uniaxial wetting layer whose thickness increases as $|\ln t|$ and whose uniaxial axis $\hat{\mathbf{n}} \perp \hat{\mathbf{k}}$.

rized by plotting $u_{s}$ and $v_{s}$ as a function of $t$ for different values of $g$. This is shown in Figs. 6(a) and 6(b). It is to be noted that for $g>g_{c}=0.12$, variation of $u_{s}$ and $v_{s}$ at $t=0$ exists only in their slopes across $t=0$, i.e., the transition is second order in nature. For $t<0$, the $\psi=\pi / 2$ bulk state is always favored, and the boundary layer is bi- axial in nature. What is striking, however, is that as $t \rightarrow 0$ from above the bulk phase in fact nucleates from the boundary layer through complete wetting. This is illustrated in Fig. 7. It is seen that at $t=10^{-5}$, there is an uniaxial section (with $\psi=\pi / 2$ so that $-u_{B} / 2, v=u_{B} / 2$ ) of the boundary layer, characterized by their flat variation as a function of $\xi$, which increases in thickness as $|\ln t|$. As $\xi$ increases beyond this section, however, the boundary layer again acquires biaxial character until at a certain point $v$ vanishes, and the boundary layer returns to being uniaxial. The uniaxial boundary-layer section eventually becomes the bulk uniaxial state as $t$ reaches 0 . The value of $g=g_{c}=0.12$ therefore separates partial wetting for $g<g_{c}$ from complete wetting at $g>g_{c}$.

Since the phenomena described above depend crucially on the magnitude of $g$, it is noted here that while $g$ is directly proportional to $G$, the substrate-nematic-liquidcrystal interaction strength, it is also inversely proportional to $B^{2}$. Therefore an alternative way of increasing $g$ is to choose liquid crystals with a weak first-order nematic-isotropic transition (small $B$ ). It should also be remarked that the phenomena predicted by the present substrate-nematic-liquid-crystal interaction differs from those resulting from a $U S^{2} \delta(z)$-type interaction [12] with a positive $U$. In that case there will also be a disordered surface layer, but instead of a negative order parameter the value of $S$ in the surface layer is positive. The experimental results shown in this work indicate that even if the $U S^{2}$-type interaction is present, it is not the dominant one.

One of us (P.S.) wishes to thank D. Allender for comments that led to helpful insights on the biaxial state. The research of T.M. and Y.R.S. was supported by the NSF Solid State Chemistry Grant No. DMR-8717137. T.M. also acknowledges support from the Department of Education.
*Present address: Institute of Physics, Academia Sinica, Beijing 100080, People's Republic of China.

[1] P. Sheng, Phys. Rev. Lett. 37, 1059 (1976).

[2] K. Miyano, Phys. Rev. Lett. 43, 51 (1979).

[3] P. Sheng, Phys. Rev. A 26, 1610 (1982).

[4] T. J. Sluckin and A. Poniewierski, in Fluid Interfacial Phenomena, edited by C. A. Croxton (Wiley, New York, 1986), Chap. 5.

[5] H. Yokoyama, J. Chem. Soc., Faraday Trans. 2, 84, 1023 (1988).

[6] H. Yokoyama, S. Kobayashi, and H. Kamei, Appl. Phys. Lett. 41, 438 (1982).
[7] W. Chen, L. J. Martinez-Miranda, H. Hsiung, and Y. R. Shen, Phys. Rev. Lett. 62, 1860 (1989).

[8] P. Sheng and E. B. Priestley, in Introduction to Liquid Crystals, edited by E. B. Priestley, P. J. Wojtowicz, and P. Sheng (Plenum, New York, 1975), Chap. 10.

[9] T. J. Sluckin and A. Poniewierski, Phys. Rev. Lett. 26, 2907 (1985).

[10] A. K. Sen and D. E. Sullivan, Phys. Rev. A 35, 1391 (1987).

[11] H. J. Coles, Mol. Cryst. Liquid Cryst. 49, 67 (1978).

[12] A. Poniewierski and T. J. Sluckin, Liquid Cryst. 2, 281 (1987). 